\title{
Caracterização das pesquisas de métodos mistos em enfermagem publicadas no Journal of Mixed Methods Research
}
Characterization of mixed methods research in nursing published in the Journal of Mixed Methods Research

Caracterización de la investigación de métodos mixtos en enfermería publicada en el Journal of Mixed Methods Research

\section{José Luís Guedes dos Santos ${ }^{\mathrm{I}}$, Greici Capellari Fabrizzio ${ }^{\mathrm{II}}$, Caroline Cechinel Peiter ${ }^{\mathrm{III}}$, Daiana de Mattia ${ }^{\mathrm{IV}}$, Daniele Perin ${ }^{\mathrm{V}}$, Elisiane Lorenzini ${ }^{\mathrm{VI}}$}

\begin{abstract}
Resumo: Objetivo: caracterizar a produção científica da área da enfermagem publicada no Journal of Mixed Methods Research. Método: estudo documental, com abordagem quantitativa. A coleta de dados foi realizada de abril a maio de 2019, a partir do termo Nursing no campo de busca da página online do periódico, considerando o recorte temporal de 2007 a 2018. Os dados foram analisados por meio de estatística descritiva. Resultados: identificaram-se 37 artigos originais, publicados principalmente a partir de 2011 (70,2\%), com destaque para os Estados Unidos (45,9\%). A triangulação concomitante ou paralelo-convergente foi o principal desenho metodológico adotado pelos artigos (32,4\%). Nas etapas qualitativa e quantitativa das pesquisas, predominaram estudos exploratório-descritivos (12,4\%) e transversais $(21,7 \%)$, respectivamente. Considerações finais: evidenciaram-se múltiplas possibilidades no emprego dos métodos mistos na pesquisa em enfermagem. Os resultados do estudo poderão auxiliar pesquisadores interessados na utilização de métodos mistos de pesquisa.
\end{abstract}

Descritores: Enfermagem; Pesquisa em enfermagem; Metodologia; Pesquisa metodológica em enfermagem

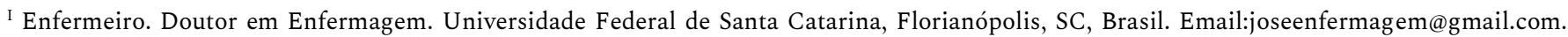
ORCID: http://orcid.org/0000-0003-3186-8286

${ }^{\text {II }}$ Enfermeira. Mestre em Enfermagem. Universidade Federal de Santa Catarina, Florianópolis, SC, Brasil. Email: greicicapellari@hotmail.com. ORCID: http://orcid.org/0000-0002-3848-5694

III Enfermeira. Mestre em Enfermagem. Universidade Federal de Santa Catarina, Florianópolis, SC, Brasil. Email: carolcechinel@gmail.com. ORCID: http://orcid.org/0000-0003-0032-6791

IV Enfermeira. Mestre em Gestão do Cuidado de Enfermagem. Universidade Federal de Santa Catarina, Florianópolis, SC, Brasil. Email: daimattia@gmail.com. ORCID: http://orcid.org/0000-0003-0301-2130

v Enfermeira. Mestre em Gestão do Cuidado de Enfermagem. Universidade Federal de Santa Catarina, Florianópolis, SC, Brasil. Email: danyperin@gmail.com. ORCID: http://orcid.org/0000-0002-2868-7719

VI Enfermeiro. Doutora em Enfermagem. Universidade Federal de Santa Catarina, Florianópolis, SC, Brasil. Email: elisilorenzini@gmail.com. ORCID: https://orcid.org/0000-0001-8426-2080
} 


\begin{abstract}
Objective: to characterize the scientific production in the field of nursing published in the Journal of Mixed Methods Research. Method: documentary study, with a quantitative approach. Data collection was carried out from April to May 2019, using the term Nursing in the search field of the journal's online page, considering the time frame from 2007 to 2018. The data were analyzed using descriptive statistics. Results: 37 original articles were identified, published mainly since 2011 (70.2\%), with emphasis on the United States (45.9\%). Concomitant or parallel-convergent triangulation was the main methodological design adopted by the articles (32.4\%). In the qualitative and quantitative stages of research, exploratory-descriptive (12.4\%) and cross-sectional (21.7\%) studies predominated, respectively. Final considerations: there were multiple possibilities in the use of mixed methods in nursing research. The results of the study may help researchers interested in the use of mixed research methods.
\end{abstract}

Descriptors: Nursing; Nursing research; Methodology; Nursing methodological research

Resumen: Objetivo: caracterizar la producción científica de enfermería publicada en el Journal of Mixed Methods Research. Método: estudio documental, con enfoque cuantitativo. La recopilación de datos se llevó a cabo de abril a mayo de 2019, utilizando el término Enfermería en el campo de búsqueda de la página en línea de la revista, considerando el período de tiempo de 2007 a 2018. Los datos se analizaron mediante estadísticas descriptivas. Resultados: Se identificaron 37 artículos originales, publicados principalmente a partir de 2011 (70,2\%), con énfasis en los Estados Unidos (45,9\%). La triangulación concomitante o convergente en paralelo fue el diseño metodológico principal adoptado por los artículos $(32,4 \%)$. En las etapas cualitativa y cuantitativa de la investigación, predominaron los estudios exploratorios descriptivos (12.4\%) y transversales (21.7\%), respectivamente. Consideraciones finales: había múltiples posibilidades en el uso de métodos mixtos en la investigación de enfermería. Los resultados del estudio pueden ayudar a los investigadores interesados en el uso de métodos de investigación mixtos.

Descriptores: Enfermería; Investigación en enfermería; Metodología; Investigación metodológica en enfermería.

\title{
Introdução
}

Os estudos de métodos mistos apresentam características singulares na coleta, análise e combinação de abordagens de pesquisa quantitativas e qualitativas em um mesmo desenho de pesquisa. O pressuposto central para o emprego desse método é de que a interação entre a pesquisa quantitativa e qualitativa fornece possibilidades de análises mais robustas. ${ }^{1}$

A origem desse método de pesquisa é relativamente recente, sendo que a sua descrição se deu com maior ênfase no início dos anos 2000, especialmente com a publicação do livro denominado Foundations of Mixed Methods Research: Integrating Qualitative and Quantitatives. ${ }^{2}$ Entretanto, na década de 1960 e 1970, já se discutia a possibilidade de misturar métodos entre antropólogos e sociólogos, o que originou o termo “triangulação”. No decorrer da década de 1980, a pesquisa de métodos mistos apresentou um crescimento discreto, com o 
surgimento dos primeiros desenhos de estudo mencionando a mixagem de dados. Assim, observa-se que o desenvolvimento de grande parte da literatura na área de métodos mistos de pesquisa ocorreu no final da década de $1990 .^{3}$

Nesse contexto de expansão dos métodos mistos, John W. Creswell tem se destacado, internacionalmente, como um grande metodologista nessa área. Conhecido no Brasil como Creswell, é um pesquisador norte-americano que tem se dedicado à demonstração de estratégias e desenvolvimento de desenhos de pesquisa em sua plena empregabilidade mista, passíveis de aplicação nas mais diversas áreas do conhecimento. ${ }^{4}$ Conforme esse autor, métodos mistos é uma abordagem de pesquisa ou metodologia focada nas questões de investigação que englobam a compreensão do contexto pesquisado. Além disso, influências culturais também podem ser captadas, considerando-se uma perspectiva multinível, enquadrando o processo investigativo dentro de posicionamentos teóricos e filosóficos. ${ }^{5}$

Para isso, devem ser empregados rigorosos métodos de pesquisa quantitativa, quando a pretensão é avaliar a magnitude e a frequência de dado objeto de investigação, e, da mesma forma, empregar rigorosos métodos de pesquisa qualitativa, para explorar, em profundidade, o significado e a compreensão de dado objeto ou fenômeno em estudo entre os participantes. Além disso, é possível aplicar múltiplas técnicas, tais como: entrevistas em profundidade e coleta de dados quantitativos em estudos de intervenção, o que possibilita a integração ou combinação intencional dos métodos quantitativos e qualitativos. Dessa forma, buscar-se-ão os pontos fortes de cada abordagem, minimizando, portanto, as fragilidades inerentes a cada um dos métodos quando são empregados de forma isolada. ${ }^{3-5}$

Nos métodos mistos, a questão de pesquisa orienta: a coleta e a análise dos dados; a integração dos dados, a qual poderá ser concomitante ou não; a definição sobre qual abordagem, entre a quantitativa ou qualitativa, será tratada como prioridade no estudo ou se ambas receberão o mesmo peso; e, a definição sobre realizar esse procedimento em uma única pesquisa ou em 
múltiplas fases de um estudo maior. Essas decisões são tomadas sempre considerando as visões filosóficas e lentes teóricas, as quais são fundamentais e auxiliam nas reflexões para tomada de decisão sobre os momentos cruciais que permitem combinar os bancos de dados, bem como, subsidiam os passos a serem dados, de acordo com o plano para a condução do estudo. ${ }^{1}$

No campo da saúde, a pesquisa de métodos mistos tem sido utilizada para avaliar o desenvolvimento e a incorporação na prática de intervenções e ensaios clínicos, observar a resposta do paciente ou a percepção dos profissionais quanto a programas implementados, assim como, em estudos que objetivam verificar a relação entre fatores comportamentais e sociais e os resultados específicos de saúde. ${ }^{6} \mathrm{Na}$ área de enfermagem, os estudos de métodos mistos ainda são recentes, porém representam uma tendência de crescimento à medida que podem ampliar as interpretações/inferências das pesquisas, propiciando um olhar sobre o cuidado em suas diferentes perspectivas. Os estudos com métodos mistos diferenciam-se pela elaboração das metainferências, ou seja, inferências aprofundadas sobre fenômenos complexos de pesquisa, que são geradas ou emergem da análise combinada de dados quantitativos e qualitativos. ${ }^{4}$

A expansão do uso de métodos mistos, tanto na área da saúde e enfermagem quanto nos demais campos de conhecimento, tem estimulado a publicação de livros, artigos e números especiais sobre essa abordagem metodológica. Nesse contexto, foi lançado, em 2007, o Journal of Mixed Methods Research (JMMR), uma revista científica internacional destinada, especificamente, à publicação de artigos empíricos, metodológicos e teóricos sobre a pesquisa de métodos mistos oriundas das ciências sociais, humanas e da saúde. O periódico é vinculado à Mixed Methods International Research Association (MMIRA) e alcançou, em 2018, o fator de impacto de 3.524, ficando em $1^{\text {o }}$ lugar no ranking das revistas da área de ciências sociais e interdisciplinar. ${ }^{7}$

O destaque do JMMR na socialização do conhecimento sobre métodos mistos e a necessidade de compreender como a enfermagem tem aplicado essa metodologia na sua produção científica justificam a realização deste estudo. Assim, delineou-se como questão de 
5 | Santos JLG, Fabrizzio GC, Peiter CC, Mattia D, Perin D, Lorenzini E

pesquisa: quais são as características da produção científica da área da enfermagem publicada no Journal of Mixed Methods Research? Nesse sentido, o objetivo deste estudo foi caracterizar a produção científica da área da enfermagem publicada no Journal of Mixed Methods Research.

\section{Método}

Trata-se de um estudo documental, de abordagem quantitativa, realizado no sítio eletrônico do JMMR. ${ }^{7}$ A coleta de dados foi realizada entre abril e maio de 2019. O termo “Nursing” e o filtro “Artigo de Pesquisa” foram utilizados para seleção dos artigos no campo de busca da página do JMMR. Foram excluídos artigos que não apresentaram resultados de pesquisa empírica da área da enfermagem. A busca englobou todos os artigos desde 2007, ano de publicação do primeiro número do periódico, até o último número de 2018.

Durante esse período, o periódico publicou 334 artigos. A partir dos critérios supracitados, a busca inicial gerou 81 artigos, dos quais 67 estavam caracterizados como artigo de pesquisa, sendo que 62 foram publicados de 2007 a 2018. Esses artigos foram submetidos à leitura na íntegra, identificando-se que 25 não apresentavam resultados de pesquisa original. Desse modo, restaram 37 estudos, os quais foram incluídos na análise. Ressalta-se que o JMMR é um periódico eletrônico e que todos os artigos estavam disponíveis on-line na integra, mediante acesso via Portal de Periódicos da Coordenação de Aperfeiçoamento de Pessoal de Nível Superior (CAPES).

Os artigos foram organizados e tabulados com auxílio do software Excel $^{\circledR}$, considerando as seguintes variáveis: ano; número de autores; país de origem; Instituição de Ensino Superior (IES) dos autores; palavras-chave; objetivo; método; técnicas de coleta de dados; participantes; cenário da pesquisa; se apresentava estratégia adotada explícita no artigo; tipo de combinação dos dados; apresentação de diagrama ilustrando o desenho da pesquisa, e, vantagens/contribuições do uso de métodos mistos para a pesquisa. Para análise do país de 
origem e IES, foram consideradas as informações referentes ao primeiro autor de artigo. Os dados foram analisados por meio de estatística descritiva.

Esta pesquisa não foi submetida à apreciação de Comitê de Ética em Pesquisa com Seres Humanos, uma vez que foram utilizados dados disponíveis online, via portal de periódicos da Coordenação de Aperfeiçoamento de Nível Superior. Porém, foram respeitados todos os aspectos éticos referentes à citação e autoria dos estudos consultados.

\section{Resultados}

Do total de 37 artigos, a maioria era oriunda dos Estados Unidos ( $\mathrm{n}=17 ; 46 \%$ ). Quanto ao ano, a maior quantidade de publicações foi verificada no ano de 2011 ( $\mathrm{n}=6 ; 16,3 \%)$. A Tabela 1 apresenta a relação completa dos artigos conforme ano e país de origem.

Tabela 1 - Classificação dos artigos da área da enfermagem publicados no JMMR, conforme ano e país de origem. Florianópolis, 2019.

\begin{tabular}{llc}
\hline Variável & $\mathbf{n}(\%)$ \\
\hline Ano & 2007 & $2(5,4 \%)$ \\
2008 & $5(13,5 \%)$ \\
2009 & $2(5,4 \%)$ \\
2010 & $2(5,4 \%)$ \\
2011 & $6(16,3 \%)$ \\
2012 & $3(8,1 \%)$ \\
2013 & $5(13,5 \%)$ \\
2014 & $3(8,1 \%)$ \\
2015 & $5(13,5 \%)$ \\
2016 & $3(8,1 \%)$ \\
2017 & $1(2,7 \%)$ \\
\hline País & Estados Unidos & $17(46 \%)$ \\
Reino Unido & $6(16,2 \%)$ \\
Canadá & $3(8,1 \%)$ \\
Austrália & $2(5,4 \%)$ \\
África do Sul & $1(2,7 \%)$ \\
China & $1(2,7 \%)$ \\
Índia & $1(2,7 \%)$ \\
Irã & $1(2,7 \%)$ \\
Irlanda & $1(2,7 \%)$ \\
Israel & $1(2,7 \%)$ \\
Nova Zelândia & $1(2,7 \%)$ \\
Holanda & $1(2,7 \%)$ \\
Suíça & $1(2,7 \%)$ \\
\hline
\end{tabular}


Em relação à autoria dos artigos, o número de autores variou de um a dez, sendo que prevaleceu a coautoria de três autores ( $n=20 ; 54,1 \%)$. Quanto às palavras-chave, foram identificados 169 termos diferentes. A palavra-chave usada com maior frequência foi "estudo misto" ou "pesquisa de métodos mistos" ( $\mathrm{n}=21 ; 12,4 \%)$. Constatou-se, ainda, recorrência dos termos HIV/AIDS ( $\mathrm{n}=5 ; 3,0 \%)$ e violência $(\mathrm{n}=2 ; 1,2 \%)$.

Quanto ao objetivo dos estudos, $14(37,8 \%)$ eram estudos metodológicos com foco descrição do desenvolvimento da pesquisa de método misto. Para isso, enfocavam principalmente a apresentação dos procedimentos metodológicos e, em segundo plano, a descrição dos resultados empíricos da pesquisa.

No tangente ao desenho da pesquisa, na etapa qualitativa, o método mais usado foi o desenho exploratório-descritivo $(\mathrm{n}=4 ; 12,4 \%)$, seguido da Teoria Fundamentada nos Dados $(\mathrm{n}=3$; 9,3\%). Cinco artigos (15,9\%) adotaram outros desenhos qualitativos e dois artigos (6,2\%) citaram o uso da análise de conteúdo na etapa qualitativa. Na etapa quantitativa, os estudos transversais foram o método mais prevalente $(n=7 ; 21,7 \%)$, seguido de estudos de validação de instrumentos $(n=3 ; 9,3 \%)$ e longitudinal $(n=2 ; 6,2 \%)$. Também foi evidenciado o estudo de caso, método que pode ser empregado tanto em pesquisa qualitativa quanto quantitativa $(3,1 \%)$.

Em relação aos instrumentos utilizados para coleta de dados, 19 artigos (51,3\%) relatam a aplicação de questionários e entrevistas semiestruturadas. Cinco estudos (13,5\%) citaram a utilização de entrevistas e grupo focal. Além disso, cinco pesquisas $(13,5 \%)$ adotaram somente entrevistas.

No que tange aos participantes das pesquisas, pacientes estiveram presentes em 13 $(35,1 \%)$ estudos, enquanto profissionais de saúde foram incluídos em dez $(27,0 \%)$ artigos, usuários do sistema de saúde e membros da sociedade foram considerados em oito (21,6\%), cuidadores em cinco $(13,5 \%)$ e estudantes de graduação/pós-graduação em quatro $(10,8 \%)$ 
Caracterização das pesquisas de métodos mistos em enfermagem publicadas no.... $\mid 8$

estudos. $\mathrm{Na}$ etapa qualitativa, o número de participantes variou de seis a 308 . $\mathrm{Na}$ etapa quantitativa, identificou-se uma variação de 16 a 2.444 sujeitos.

A estratégia adotada pelos autores estava explícita em $23(62,2 \%)$ artigos, sendo 12 $(32,4 \%)$ do tipo triangulação concomitante ou paralelo-convergente, seis $(16,2 \%)$ explanatória sequencial e cinco (13,5\%) exploratória sequencial. O tipo de combinação dos dados adotado na pesquisa foi especificado em $15(40,5 \%)$ dos artigos, dos quais $12(32,4 \%)$ indicaram a realização de combinação por integração, dois $(5,4 \%)$ por incorporação e um $(2,7 \%)$ por conexão. Em relação à apresentação da metodologia, 15 (40,5\%) artigos fizeram uso de diagramas, sumarizando as etapas de coleta e análise de dados, a estratégia utilizada e o tipo de combinação adotado. Em todos os artigos selecionados, houve a descrição de vantagens e contribuições do uso de métodos mistos em pesquisas em enfermagem, conforme apresentado na Tabela 2.

Tabela 2 - Vantagens e contribuições na utilização de métodos mistos, declaradas em pesquisas da área de enfermagem publicadas no JMMR. Florianópolis, 2019.

\begin{tabular}{|c|c|}
\hline Vantagens e contribuições & $\mathbf{n}(\%)$ \\
\hline $\begin{array}{l}\text { Conhecimento ampliado da temática, a partir de múltiplas perspectivas para } \\
\text { compreensão de fenômenos complexos }\end{array}$ & $15(40,5 \%)$ \\
\hline $\begin{array}{l}\text { Superação de fragilidades de um único método e produção de dados mais } \\
\text { aprofundados em comparação ao uso de uma abordagem isolada }\end{array}$ & $9(24,3 \%)$ \\
\hline $\begin{array}{l}\text { Ampliação do conhecimento em áreas substantivas emergentes ou ainda pouco } \\
\text { exploradas, com vistas à identificação de necessidades de intervenção }\end{array}$ & $7(18,9 \%)$ \\
\hline $\begin{array}{l}\text { Associação e comparação dos dados conferem maior confiabilidade/validade à } \\
\text { pesquisa }\end{array}$ & $3(8,1 \%)$ \\
\hline $\begin{array}{l}\text { Construção de equipes de pesquisa interdisciplinares, a partir do envolvimento de } \\
\text { participantes que contribuem para elaboração de compreensões mais aprofundadas e } \\
\text { soluções para problemas complexos }\end{array}$ & $3(8,1 \%)$ \\
\hline
\end{tabular}




\section{Discussão}

Ao analisar o ano de publicação dos artigos da área da enfermagem publicados no JMMR, percebe-se que houve oscilações quanto ao número de publicações ao longo do período analisado. Porém, pode-se considerar que as publicações se tornaram mais constantes, principalmente a partir de 2011. Esse fato pode ser sustentado pelo interesse no desenvolvimento de pesquisas de métodos mistos, o qual tem aumentado nos últimos anos. ${ }^{1}$ Isso se deve à fase de defesa e expansão deste método, na qual emergiram muitos autores que hoje defendem a pesquisa de métodos mistos como uma metodologia, estendo-a a muitas disciplinas, em vários países.

Em relação ao país de origem dos autores, os Estados Unidos apresentaram destaque na concentração de autores/pesquisadores que utilizam métodos mistos como delineamento dos seus estudos. Praticamente, a metade dos artigos analisados é proveniente desse país. Pode-se fazer uma relação dessa ocorrência ao autor John Creswell, autor norte-americano conhecido pelo emprego do método, com muitos livros escritos sobre a temática. Além disso, Creswell é um difusor do método, portanto, pressupõe-se que isto contribua para o interesse do desenvolvimento de estudos dessa natureza no país. ${ }^{4}$ Os Estados Unidos também são reconhecidos pela sua representatividade na concentração de produção do conhecimento científico no mundo. ${ }^{8}$ Como 37 (78,3\%) dos artigos são originários da América do Norte e Europa, considera-se importante a ampliação de estratégias de divulgação e visibilidade da pesquisa de métodos mistos em outros cenários do globo.

Ao se considerar a coautoria nos artigos, mais da metade apresentam menos de três autores. Esta afirmativa pode estar associada ao fato de que boa parte das pesquisas são de âmbito local, ou seja, não se constituem em estudos multicêntricos com grande número de participantes ou fases na etapa qualitativa. É importante pontuar que o desenvolvimento de uma pesquisa de métodos mistos demanda planejamento para que todas as etapas sejam realizadas 
Caracterização das pesquisas de métodos mistos em enfermagem publicadas no.... $\mid 10$

com qualidade e em consonância com o rigor que toda pesquisa requer, além de respeitar os princípios éticos. Além disso, para zelar pela qualidade dos dados coletados, muitas vezes, é necessário estruturar uma equipe de pesquisa, que contemple integrantes com expertise em estudos qualitativos e quantitativos, que podem auxiliar na coleta de dados, contudo, sempre sob supervisão do pesquisador principal. ${ }^{3}$

Quanto às palavras-chave, houve indicação de que se tratava de estudos de métodos mistos, porém não houve menção de uma determinada área específica da enfermagem. Nesse sentido, é importante ressaltar que a pesquisa de métodos mistos pode ser aplicada a uma ampla variedade de áreas dentro das ciências sociais e da saúde, uma vez que permite a melhor compreensão de problemas complexos., ${ }^{5,9}$ A integração dos resultados eleva o método de pesquisa para um nível que não seria alcançado simplesmente colocando juntos os resultados de pesquisas separadas, qualitativas e quantitativas, conduzidas sem total atenção à integração. ${ }^{10}$

No que tange à área da enfermagem, essa abordagem metodológica pode ser empregada para compreender o aspecto multidimensional dos problemas que os enfermeiros enfrentam na prática assistencial. Ainda, para obter uma compreensão mais abrangente das circunstâncias de saúde dos pacientes, fornecendo uma explicação de sua condição, bem como, capturando suas experiências pessoais de saúde e doença. Destaca-se, também, que a aplicação desse desenho de pesquisa pode contribuir substancialmente para o desenvolvimento e a avaliação das intervenções de enfermagem. ${ }^{11}$

Os objetivos apresentados pelos estudos são heterogêneos, porém grande parte se preocupa em apresentar os aspectos metodológicos do método misto, justificando o seu uso como uma ferramenta para ampliação do conhecimento e busca de evidências que elucidem o fenômeno estudado. Esse tipo de artigo pode ser caracterizado como metodológico, pois não visava à apresentação em profundidade dos resultados empíricos da pesquisa, como um artigo original. Estudos dessa natureza buscam mostrar a utilização da abordagem metodológica, no 
11 | Santos JLG, Fabrizzio GC, Peiter CC, Mattia D, Perin D, Lorenzini E

sentido de orientar os pesquisadores na compreensão e aplicação do método e consequentemente para contribuir na sua consolidação. ${ }^{3}$ Além disso, esse tipo de objetivo pode ser justificado pelo caráter metodológico do $\mathrm{JMMR}^{12}$ o qual possui como requisito para a submissão e publicação de artigo, que o mesmo exponha, claramente, qual a contribuição teórica ou metodológica do mesmo para a área de pesquisa de métodos mistos.

A definição das estratégias de pesquisa que são associadas em um estudo de métodos mistos está diretamente relacionada à questão de pesquisa, ao escopo da investigação e seus objetivos. Embora a literatura sugira que na pesquisa de métodos mistos as metodologias podem ser descritas e rotuladas, cada uma pertence a uma abordagem diferente e podem ser combinadas. ${ }^{13}$

A técnica de coleta de dados mais utilizada foi entrevista e aplicação de questionários. Nos estudos quantitativos, priorizam-se técnicas objetivas de coleta, para que as informações possam ser quantificadas e estruturadas de forma a comparar os mesmos dados de todos os participantes. Em pesquisas qualitativas, a entrevista é a mais comum, porém também são descritas outras, como a observação, documentos, artefatos e fotografias. A entrevista é uma técnica comumente utilizada para compreender questões amplas de pesquisa, que vão sendo afuniladas à medida que a entrevista progride. Uma entrevista exige habilidade do pesquisador para conduzir e estimular o participante a falar abertamente sobre a temática em voga. ${ }^{14}$

Os participantes de pesquisa incluídos nos estudos analisados apresentaram-se de forma heterogênea. Se constituíram como sujeitos de pesquisa, pacientes e usuários, profissionais, professores, estudantes e outros membros da sociedade, indicando o interesse na exploração de grupos distintos e complementares na efetivação das práticas de cogestão, preconizadas no compartilhamento de responsabilidades no alcance de objetivos em saúde. ${ }^{15}$

A triangulação concomitante, também chamada de paralelo-convergente, foi o desenho que prevaleceu nos estudos encontrados. Essa abordagem compreende execução simultânea do 
estudo quantitativo e qualitativo. Tem como principal vantagem a economia de tempo para o pesquisador, o que pode justificar o destaque do seu uso nas pesquisas de métodos mistos na área da enfermagem. Cabe ressaltar que a estratégia paralelo-convergente indica igualdade de pesos entre as etapas quantitativa e qualitativa mixadas, e busca fazer com que estas se complementem mutuamente por meio de integração dos dados. ${ }^{1,5}$

O diferencial da pesquisa de métodos mistos, quando em comparação a um projeto quali-quantitativo, acontece na combinação dos dados. O tipo de combinação adotado vai depender se o uso da pesquisa mista tem por objetivo identificar se os dados qualitativos são válidos para toda a população, aprofundar questões específicas que tenham se destacado em entrevistas fechadas ou questionários, ou ainda se tem o intuito de complementar mutuamente os resultados das diferentes metodologias. Quando o objetivo é verificar se os resultados de uma pesquisa qualitativa, com um pequeno grupo de participantes, se repetiriam ou se seriam válidos para toda a população, por exemplo, o pesquisador pode optar, primeiro, por procurar entender os caminhos pelos quais um fenômeno específico se desenvolve, usando entrevistas narrativas. Na sequência, pode investigar se esses caminhos são válidos para a população em geral, usando um survey. ${ }^{13}$

O tipo de combinação mais frequente nos estudos analisados foi a integração, o que denota coerência com a estratégia mais prevalente, paralelo-convergente, uma vez que o uso desta estratégia pressupõe que a combinação dos dados seja feita por integração. Nesse sentido, reforça-se que a compreensão dos objetos e fenômenos do estudo ao final da pesquisa mista perpassa necessariamente a interpretação de forma conjunta dos resultados quantitativos e qualitativos da pesquisa. ${ }^{16}$

Para maior difusão de métodos mistos nas pesquisas em enfermagem e saúde, os pesquisadores experientes podem ser encorajados a lançar mão de algumas estratégias visando à disseminação do método, tais como: integrar pesquisadores iniciantes em suas equipes de 
pesquisa ao desenvolverem projetos de métodos mistos e realizar mentoria em equipe. Essa orientação, quando bem-sucedida, pode se constituir em um meio para resultar em progresso acadêmico; aumento da produtividade em pesquisa; definição de carreira, permanência e boa relação no trabalho. Essas ações de colaboração possibilitam oportunidades de aprendizado mútuo, em conformidade com os preceitos epistemológicos dos métodos mistos. ${ }^{17}$

\section{Considerações finais}

Este trabalho permitiu a caracterização das produções da área de enfermagem publicadas em um periódico especializado em métodos mistos, o JMMR. Constatou-se que a maioria dos artigos foi publicada a partir de 2011(70,2\%), com destaque para os Estados Unidos (45,9\%). A triangulação concomitante ou paralelo-convergente foi o desenho adotado por grande parte dos estudos $(32,4 \%)$. Nas etapas qualitativa e quantitativa das pesquisas, predominaram estudos exploratório-descritivos (12,4\%) e transversais $(21,7 \%)$, respectivamente.

Assim, evidenciaram-se múltiplas possibilidades no emprego dos métodos mistos e o esforço dos autores na busca por avanços no emprego dos métodos mistos na pesquisa em enfermagem. Porém, destaca-se a importância de ações de divulgação e maior visibilidade da pesquisa de métodos mistos como estratégia metodológica, principalmente no contexto latinoamericano.

Embora esta pesquisa tenha sido desenvolvida em sítio eletrônico de abrangência internacional, seus resultados são limitados a um único periódico científico. Ainda assim, considera-se que os achados do presente estudo fornecem subsídios para pesquisadores da área da saúde e enfermagem, que poderão empregá-los no delineamento de suas futuras investigações. 
Caracterização das pesquisas de métodos mistos em enfermagem publicadas no.... $\mid 14$

\section{Referências}

1. Creswell JW, Clark VLP. Pesquisa de métodos mistos. 2ª ed. Porto Alegre (RS): Penso; 2013.

2. Pelto PJ. What is so new about mixed methods? Qual Health Res [Internet]. 2015 [acesso em 2019 out 31];25(6):734-45. Disponível

em: https://journals.sagepub.com/doi/abs/10.1177/1049732315573209?journalCode=qhra

3. Santos JLG, Erdmann AL, Meirelles BHS, Lanzoni GMM, Cunha VP, Ross R. Integração entre dados quantitativos e qualitativos em uma pesquisa de métodos mistos. Texto \& Contexto Enferm [Internet]. 2017 [acesso em 2019 out 31];26(3):e1590016. Disponível em: http://www.scielo.br/scielo.php?script=sci_arttext\&pid=S0104-07072017000300330\&lng=en

4. Oliveira JLC, Magalhães AMM, Matsuda LM. Métodos mistos na pesquisa em enfermagem: possibilidades de aplicação à luz de Creswell. Texto \& Contexto Enferm [Internet]. 2018 [acesso em 2019 out 31];27(2):e0560017. Disponível

em:

http://www.scielo.br/scielo.php?script=sci_arttext\&pid=S0104-07072018000200323\&lng=en

5. Creswell JW. Projeto de pesquisa: métodos qualitativo, quantitativo e misto. $3^{\mathbf{a}}$ ed. Porto Alegre (RS): Artmed; 2010.

6. Guettermana TC, Creswell JW, Deutschb C, Galloc JJ. Skills development and academic productivity of scholars in the nih mixed methods research training program for the health sciences. Int J Mult Res Approaches [Internet]. 2018 [acesso em 2019 out 31];10(1):373-89. Disponível em: https://www.ncbi.nlm.nih.gov/pmc/articles/PMC6394873/

7. SAGE Publications. Journal of Mixed Methods Research [Internet]. 2020 [acesso em 2019 jun 26 ]. Disponível em: https://journals.sagepub.com/journal-info/mmr

8. Costa MC, Silva RGL. A dinâmica do conhecimento biomédico e em saúde: uma interpretação sociológica. Sociologias [Internet]. 2019 [acesso em 2019 out 31];21(50):18-47. Disponível em: http://www.scielo.br/scielo.php?script=sci_arttext\&pid=S1517-45222019000100018

9. Galvão MCB, Pluye P, Ricarte ILM. Métodos de pesquisa mistos e revisões de literatura mistas: conceitos, construção e critérios de avaliação. InCID Rev Ciênc Inf Doc [Internet]. 2017 [acesso em 2019 out 31];8(2):4-24. Disponível em: http://www.revistas.usp.br/incid/article/view/121879

10. Lorenzini E. Pesquisa de métodos mistos nas ciências da saúde. Rev Cuid [Internet]. 2017 [acesso em 2019 out 31];8(2):1549-60. Disponível em: https://revistacuidarte.udes.edu.co/index.php/cuidarte/article/view/406/780

11. Fàbregues S, Paré MH. Appraising the quality of mixed methods research in nursing: a qualitative case study of nurse researchers' views. Nurs Inq [Internet]. 2018 [acesso em 2019 out 31];25(4):e12247. Disponível em: https://www.ncbi.nlm.nih.gov/pubmed/29927008 
12. Robinson AL, Emden CG, Croft TD, Vosper GC, Elder JA, Stirling C, et al. Mixed methods data collection in dementia research: a "progressive engagement" approach. J Mix Methods Res [Internet]. 2011 [acesso em 2019 out 31];5(4):330-44. Disponível em: https://journals.sagepub.com/doi/abs/10.1177/1558689811416940?journalCode=mmra

13. Schoonenboom J. Designing mixed methods research by mixing and merging methodologies: a 13step model. Am Behav Sci [Internet]. 2018 [acesso em 2019 out 31];62(7):998-1015. Disponível em: https://journals.sagepub.com/doi/abs/10.1177/0002764218772674? journalCode=absb

14. Polit DF, Beck CT. Fundamentos de pesquisa em enfermagem. 9aㅗ ed. Porto Alegre (RS): Artmed; 2019 .

15. McGrath C, Palmgren PJ, Liljedahl M. Twelve tips for conducting qualitative research interviews. Med Teacher [Internet]. 2018 [acesso em 2019 nov 23];41(9):1002-6. Disponível em: https://www.tandfonline.com/doi/full/10.1080/0142159X.2018.1497149

16. Oliveira JLC, Magalhães AMM, Nishiyama JAP, Vituri DW, Marcon SS, Matsuda LM. Pesquisa com métodos mistos na enfermagem: experiência na pós-graduação. Rev Enferm UFSM [Internet]. 2019 [acesso em 2019 nov 23];9(e2):1-15. Disponível em: https://periodicos.ufsm.br/reufsm/article/view/38441

17. Munce SEP, Archibald MM. The future of mixed methods: a five year projection to 2020: an early career perspective. J Mix Methods Res [Internet]. 2016 [acesso em 2019 out 31];1:11-14. Disponível em: https://journals.sagepub.com/doi/10.1177/1558689816676659

\section{Autor correspondente}

José Luís Guedes dos Santos

E-mail: joseenfermagem@gmail.com

Endereço: Universidade Federal de Santa Catarina Centro de Ciências da Saúde. Departamento de Enfermagem, sala 304. Bairro: Trindade, Florianópolis, SC.

CEP: $88040-970$

\section{Contribuições de Autoria}

\section{1 - José Luís Guedes dos Santos}

Concepção e planejamento do projeto de pesquisa, obtenção ou análise e interpretação dos dados, redação e revisão crítica.

\section{2 - Greici Capellari Fabrizzio}

Concepção e planejamento do projeto de pesquisa, obtenção ou análise e interpretação dos dados, redação e revisão crítica.

\section{3 - Caroline Cechinel Peiter}

Contribuições: concepção e planejamento do projeto de pesquisa, obtenção ou análise e interpretação dos dados, redação e revisão crítica. 
Caracterização das pesquisas de métodos mistos em enfermagem publicadas no.... $\mid 16$

\section{4 - Daiana De Mattia}

Concepção e planejamento do projeto de pesquisa, obtenção ou análise e interpretação dos dados, redação e revisão crítica.

\section{5 - Daniele Perin}

Concepção e planejamento do projeto de pesquisa, obtenção ou análise e interpretação dos dados, redação e revisão crítica.

\section{6 - Elisiane Lorenzini}

Análise e interpretação dos dados, redação e revisão crítica.

\section{Como citar este artigo}

Santos JLG, Fabrizzio GC, Peiter CC, Mattia D, Perin D, Lorenzini E. Caracterização das pesquisas de métodos mistos em enfermagem publicadas no Journal of Mixed Methods Research. Rev. Enferm. UFSM. 2019 [Acesso em: Anos Mês Dia]; vol.9 e: 1-16. DOI:https://doi.org/10.5902/2179769241298 